\title{
Advances in Scattered Electron Intensity Distribution Imaging for Microstructural Visualization and Correlations with EBSD Measurements
}

\author{
Matthew M. Nowell ${ }^{1}$, Stuart I. Wright ${ }^{1}$, Travis Rampton ${ }^{2}$ and René de Kloe ${ }^{3}$ \\ 1. EDAX Inc., Draper UT USA \\ 2. EDAX Inc., Mahwah NJ USA \\ 3. EDAX B.V., Tilburg The Netherlands
}

Electron backscatter diffraction (EBSD) has evolved into a well-established tool for microstructural characterization by providing quantitative information on crystallographic orientation and phase content and distribution. The rapid acceptance of EBSD as an analytical technique has been driven, in part, by the highly informative contrasts used to create different micrographs which effectively describe the microstructure of the material, including qualitative contrasts such as EBSD image quality measurements [1].

Even with advances in high-speed EBSD camera systems, EBSD is often described, correctly or not, as a slower analytical imaging technique, particularly for more difficult samples such as highly deformed or multi-phase geological materials. In certain situations a quick visualization of the microstructure is sufficient. One approach in this scenario is to use a Forward Scatter Detector (FSD) imaging system to provide qualitative orientation, atomic number, and topographic contrast information [2]. With FSD imaging, one or more solid-state diodes are positioned as electron detectors around the perimeter of an EBSD phosphor screen. These detectors are used to image changes in detected electron intensity. However each diode signal requires amplification and analog to digital conversion circuitry effectively limiting the number of active signals that can be processed and imaged simultaneously.

An alternative technique has been developed termed PRIAS, or Pattern Region of Interest Analysis System, which utilizes defined regions of interest (ROIs) on the EBSD detector phosphor screen as positional electron detectors to replicate traditional FSD imaging and provide new additional imaging contrasts through multiple and variable ROI positioning. In one approach, a high-speed EBSD camera is binned to obtain fast frames rates at resolutions not useful for traditional EBSD pattern indexing but which contain useful information for microstructural imaging. Another approach pre-defines ROIs to be sampled during EBSD mapping at normal binning levels to supplement EBSD data with new imaging information. A third approach analyzes ROIs positioned on stored EBSD patterns acquired during mapping. With this approach, patterns can be processed and analyzed multiple ways to extract maximum information content contained within the detected electron intensity distribution. With these last two approaches, direct correlation with traditional EBSD orientation and phase information is possible.

With all these approaches, an array of images are available showing the contrast in detected electrons hitting the various ROIs defined on the phosphor screen. In the current implementation as many as 25 positional detectors are available. These images can also be processed together through weighted arithmetic operations and through computational comparison routines to identify regions of similar and different scattered intensities. A set of PRIAS images collected from an Inconel 600 sample are shown in Figure 1. Figure 1a was generated using 3 selected ROIs to image grain orientation contrast with grain boundary precipitate topography. Figure $1 \mathrm{~b}$ was generated using an RGB coloring scheme with 7 
selected ROIs to combine grain orientation contrast with subgrain deformation contrast. Figure 1c was generated using ROI comparison to identify and visualize the position of grain boundaries within the microstructure.

This approach can also be applied to transmission EBSD (T-EBSD), where the detected electrons pass through an electron-transparent sample prior to detection. A PRIAS image generated using an RGB coloring scheme with 6 selected ROIs from an aluminum alloy foil is shown in Figure 2. Microstructural details that were difficult to accurately resolve with EBSD across the thickness variations of the foil are imaged with this technique.

\section{References:}

[1] S.I. Wright and M.M. Nowell, Microscopy and Microanalysis 12 (2006), pp. 72-84.

[2] A.P. Day and T.E Quested, Journal of Microscopy 195 (1999), pp. 186-196.
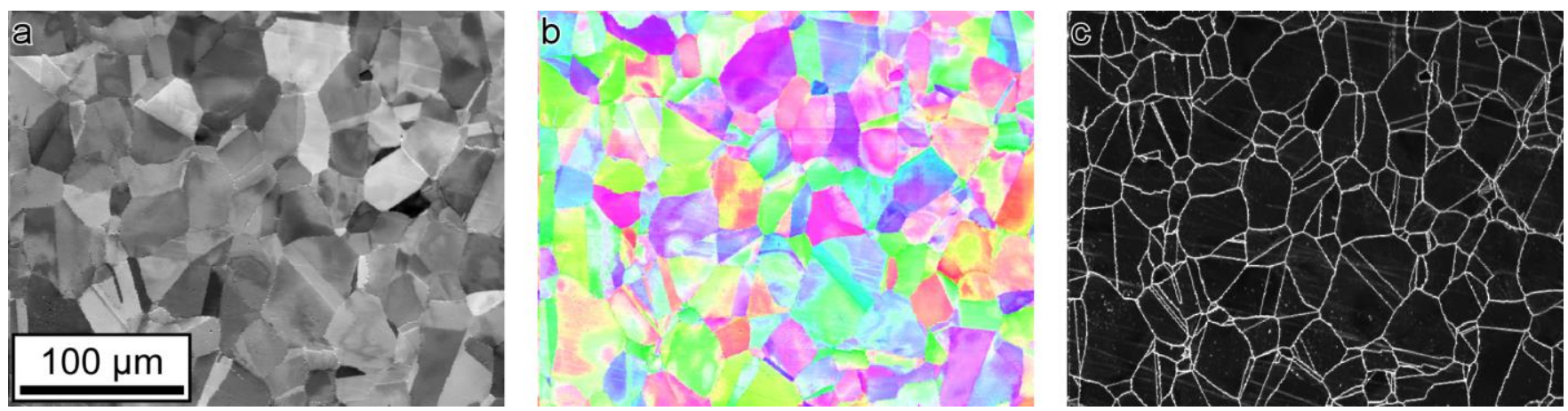

Figure 1. PRIAS images with ROIs selected to show a) orientation and grain boundary precipitate contrasts using 3 ROIs, b) orientation and deformation contrasts using 7 ROIs with an RGB coloring scheme and 3) an ROI difference map showing grain boundary position from an Inconel 600 alloy.

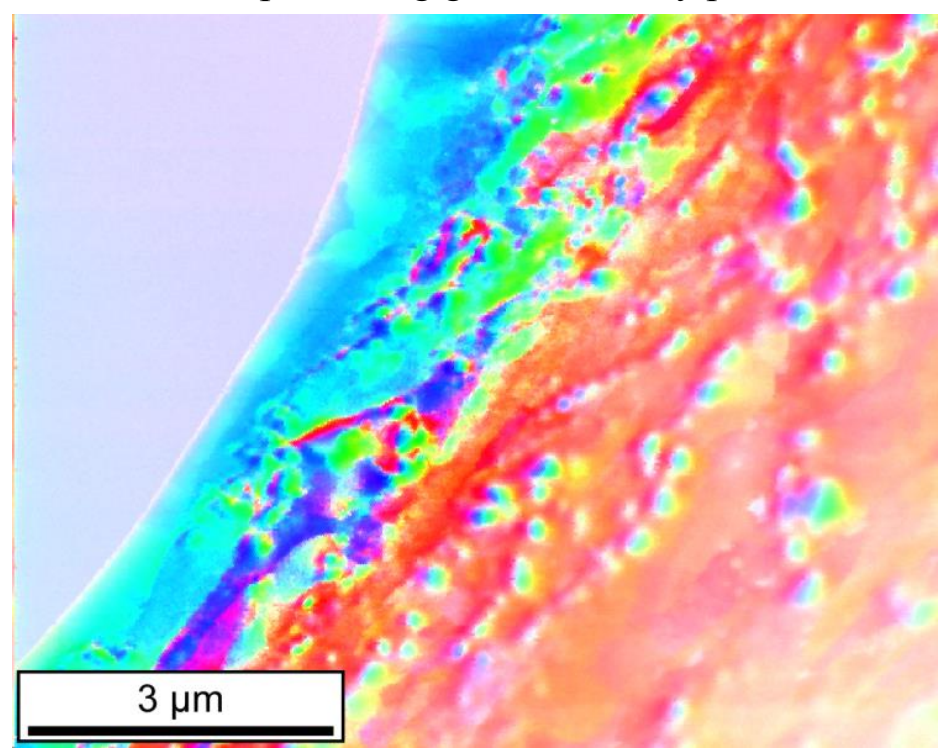

Figure 2. PRIAS image from Al T-EBSD sample using RGB color mixing with 6 selected ROIs to maximize microstructural contrasts across foil thickness. 